\title{
Bayesian Models for Deriving Biogeochemical Information from Satellite Ocean Color
}

\author{
Susanne E. Craig1, 2 and Erdem M. Karaköylü1,3
}

${ }^{1}$ Ocean Ecology Laboratory, NASA Goddard Space Flight Center, Greenbelt, Maryland, USA; susanne.e.craig@nasa.gov

${ }^{2}$ Universities Space Research Association, NASA Goddard Space Flight Center, Greenbelt, Maryland, USA

${ }^{3}$ Science Applications International Corporation, NASA Goddard Space Flight Center, Greenbelt, Maryland, USA; jkomd.283.2112@gmail.com

This manuscript is a non-peer reviewed preprint submitted to Earth ArXiv. Please note that this manuscript has yet to undergo peer review or to be formally accepted for publication in a peer reviewed journal. Please feel free to contact either of the authors - we welcome your feedback. 


\title{
Bayesian Models for Deriving Biogeochemical Information from Satellite Ocean Color
}

\author{
Susanne E. Craig ${ }^{1,2}$ and Erdem M. Karaköylü ${ }^{1,3}$ \\ ${ }^{1}$ Ocean Ecology Laboratory, NASA Goddard Space Flight Center; ${ }^{2}$ Universities Space Research Association; ${ }^{3}$ Science Applications International Corporation \\ This manuscript was compiled on November 24, 2019
}

biological oceanography | phytoplankton | machine learning | satellite remote sensing | atmospheric correction

The era of satellite ocean color began in 1978 with the launch of NASA's Coastal Ocean Color Scanner (CZCS) on board the Nimbus-7 spacecraft. Through measurement of the quantity and quality of the light reflected from the ocean, CZCS revolutionized our understanding of the intimate relationships between ocean physics and phytoplankton distribution in the world ocean (1). Generations of spaceborne sensors have subsequently followed, and satellite ocean color measurements now provide spatial and temporal distributions of phytoplankton (2) and other aquatic biogeochemical constituents (3), estimates of ocean primary productivity $(4,5)$, and have become a vital input to global models of Earth system processes and their response to a changing climate $(6,7)$.
Ocean color is derived from the signal collected at the top of the atmosphere (TOA) by a satellite spectroradiometer. The majority of this signal is due to scattering from atmospheric aerosols and reflection by wind-generated whitecaps, with only $\sim 10 \%$ maximum of the spectrum due to radiance either reflected from the ocean surface or scattered back out must, therefore, be 'subtracted' from the TOA signal in order to derive the oceanic contribution. This is achieved operationally through the process of atmospheric correction (AC), which removes the influence of sun glint, whitecaps formed by wind, and the contribution by atmospheric aerosols. This latter step takes advantage of the fact that the water body can be considered to be totally absorbing (i.e. black) in the near infrared (NIR). Any TOA signal detected at wavelengths in the NIR is then attributed to atmospheric contributions and a suitable aerosol radiance model is chosen to extrapolate to shorter wavelengths. This derived atmospheric radiance is subtracted from the total TOA spectrum, and the signal that remains is the water-leaving radiance. Remote sensing reflectance $\left(R_{r s}, s r^{-1}\right)$ i.e. the light exiting the water normalized to a hypothetical condition of an overhead Sun and no atmosphere $(8,9)$, can then be calculated. Following calculation of $R_{r s}$, various approaches are used to estimate water constituent concentrations. These fall broadly into two classes of algorithms: i) empirical band-ratio algorithms, which are derived from the statistical relationship between the ratio of two or more wavebands (blue and green) of $R_{r s}$ and in situ measurements of chlorophyll a concentration, Chl a $\left(\mathrm{mg} \mathrm{m}^{-3}\right)$, a proxy for phytoplankton biomass (2), and ii) semi-analytical through the air-water interface. The atmospheric contribution algorithms that are based on a combination of radiative transfer theory and empirically derived parameters, and that permit the retrieval of inherent optical properties (IOPs) such as spectral particulate backscattering, $b_{b p}\left(\mathrm{~m}^{-1}\right)$, and phytoplankton absorption, $a_{p h}\left(\mathrm{~m}^{-1}\right)$, coefficients that can be related to the water constituents of interest (3).

In a very general sense, $\mathrm{AC}$ approaches perform well over the open ocean where the water is totally absorbing in the NIR and the aerosol assemblage can be well modeled. However, AC performance becomes severely limited in coastal and inland waters where bottom reflectance can contaminate waterleaving signals, suspended sediments may produce a non-zero reflectance in the NIR, and/or absorbing aerosols, e.g. those generated by terrestrial anthropogenic sources (10), are difficult to model accurately. The performance of the in-water algorithms is also degraded in these regions for a variety of reasons. The band ratio algorithms were developed for use in case 1 waters, i.e. those in which ocean color is dominated by $\mathrm{Chl}$ a and all other optically active water constituents covary (11). In case 2 waters (11), where other optically active water constituents vary independently of $\mathrm{Chl}$ a (e.g. coastal waters), band ratio algorithms often perform poorly as colored dissolved organic material (CDOM) and suspended particulate material compete with phytoplankton for the absorption and scattering of blue photons, thereby confounding the algorithm's assumption of co-variability. Semi-analytical models may perform satisfactorily in case 2 waters, but model parameters such as the spectral slopes of CDOM+detrital absorption and particulate backscattering may need to be regionally tuned as their local values can vary widely (12-14). Additionally, the signal of interest may simply be swamped by competing processes - a common occurrence in case 2 waters where CDOM absorption coefficients can be an order of magnitude or greater than that of phytoplankton $(15,16)$. Finally, if AC is inaccurate, the spectral shape of the retrieved $R_{r s}$ spectrum may be distorted, meaning that the starting point for any of these ocean color models will be fundamentally flawed. As a result of these challenges, satellite measurements made over such water bodies are often unusable for quantitative studies. The loss of information from these systems is particularly egregious as they are vulnerable to climate and anthropogenic forcing (17), play host to highly productive fisheries (18), or are regions of intense atmospheric $\mathrm{CO}_{2}$ uptake (19) or sinking of organic matter for climate-relevant time scales $(20,21)$. 
Work has been devoted to improving the standard AC and a number of alternative approaches have been proposed (22). These include a multiband AC that uses multiple NIR and shortwave infrared channels (23), and several neural network (NN) techniques that provide a universal method to approximate arbitrary non-linear functions. The NNs are used to solve the AC problem directly (i.e. inputs of observed reflectance and viewing geometry and water reflectance as output), or to model the radiative transfer equation (RTE) itself, thereby saving the substantial computational time taken to solve the RTE (see Frouin et al. (22) and references therein). In a similar vein, a number of in-water studies have investigated similar approaches using NNs to estimate Chl, IOPs, apparent optical properties (AOPs) and water constituent concentrations for optically complex (i.e. case 2) waters (24-28). Craig et al. (16) developed a method based on empirical orthogonal function (EOF) analysis of hyperspectral $R_{r s}$ to estimate IOPs in an optically complex water body, then further developed the technique to estimate IOPs directly from TOA reflectance spectra, thereby combining AC with in-water IOP estimation [ref]. Collectively, these approaches present an alternative paradigm for retrieving biogeochemically relevant information from ocean color, particularly in scenarios where it may otherwise not be possible. However, ocean color science has yet to fully embrace the potential of these approaches, in particular machine learning, which has undergone rapid development and democratization. Several source codes are now available via open source platforms, and ML is used routinely and operationally in many of the Earth sciences, e.g. in meteorology (29) or plankton taxonomic studies (30), where the abundance of data enables the use of state-of-the art approaches such as deep learning. Ocean color remote sensing, on the other hand, suffers from a severe lack of labelled data (3), i.e. in situ observations that can be matched with satellite observations, and this reduces the number of approaches that can be used in a principled way to estimate IOPs and their uncertainty. Moreover, this labelled data is inherently noisy due to factors such as environmental fluctuations, measurement error, and sensor uncertainty. These obstacles make the development of generalizable models (i.e. models that capture the signal but not the noise in training data) very challenging. To address these issues, we use a Bayesian approach $(31,32)$ in developing our models. We chose this approach because the inclusion of sufficiently informed prior information can guard against overfitting, while providing transparency with respect to modeling assumptions. Furthermore, estimates of modeling and prediction uncertainty are the default of Bayesian models. Unlike frequentist concepts such as the p-value and confidence intervals, Bayesian credibility intervals derived from model posterior distributions are unambiguous and can be readily interpreted $(33,34)$.

Here, we present a machine learning effort that extends the previous work of Craig (35) by using TOA reflectance in Bayesian predictive models of the phytoplankton absorption coefficient, $a_{p h} . a_{p h}$ is an an information-rich parameter that can provide an alternative proxy of phytoplankton biomass (36), insight into community composition $(37,38)$, and can quantify the light available for photosynthesis in primary production models, making it an ideal candidate for use as an Essential Climate Variable (39) or Essential Ocean Variable (40). The ability to reliably estimate $a_{p h}$ while bypassing the considerable challenges of conventional AC in these ecologically and economically important waters provides a significant advancement of our fundamental understanding of biogeochemical processes, and the insight required to effect meaningful ecosystem management and climate change mitigation strategies.

\section{Results}

Three hierarchical Bayesian models predicting phytoplankton absorption at 6 wavebands were successfully fitted. In increasing order of complexity, these models were linear regression, linear regression with first order interaction terms, and neural network. Input variables included 6 principal components derived from 6 Rayleigh-corrected bands, in addition to a number of ancillary predictors (see Materials and Methods section). All three models were built to highlight predictor relevance. This is depicted in order of relevance for each model in the forest plots shown in Fig. 1 for phytoplankton absorption at $411 \mathrm{~nm}, a_{p h}(411)$. Note that we use $a_{p h}(411)$ in Fig. 1 as an illustrative example because this region of the spectrum is typically the most affected by inaccurate atmospheric correction and, therefore, represents the most challenging scenario for ocean color retrievals. For all models, the first three principal components appear among the more influential variables. The linear regression model identified sea surface temperature and bathymetry (sst and dep in Fig. 1, top panel) as significantly relevant in predicting $a_{p h}(411)$. For the linear regression with interaction model, interaction between the first two spectral principal components ( $p c 1$ and $p c 2)$, and interaction between the fourth principal component and the solar zenith angle ( $p c 4$ and solz) were found to be the most influential variables (Fig. 1 , middle panel). Interestingly, the neural network deemed only PC spectral information as relevant in $a_{p h}(411)$ prediction (Fig. 1, bottom panel).

The uncertainties around the relevant parameters were similar in magnitude between the two types of linear regression models. In the case of the neural network, the most relevant parameters exhibit the greatest uncertainty (Fig. 1, bottom panel), likely an effect of the small size of the data set used. This pattern changes, however, where model prediction skill is concerned. To asses each model's prediction skill, a small out-of-sample data set was used and the following criteria examined: 1) how tight the $95 \%$ credibility interval of the posterior predictive simulation was (Fig. 2); 2) where outof-sample observations occur in relation to the $95 \%$ and $50 \%$ credibility intervals (Fig. 2); and 3) how closely average predictions tracked out-of-sample observations (Figs. 2 - 5).

We found that for all bands, and for all performance criteria listed above, expected predictive performance on out-of-sample data (i.e. future, unseen data) increased with model complexity. Linear regression was the least proficient of the three models, while the Bayesian neural network was the model most likely to generalize well. Of all 6 bands tested, $a_{p h}(555)$ was the most challenging to fit across all models, likely due to the fact that phytoplankton absorption is weakest in the green spectral region. This behavior was also observed by Craig et al.(16) in their PC-based models.

\section{Discussion}

This study clearly illustrates the feasibility of retrieving the spectral phytoplankton absorption coefficient from op- 

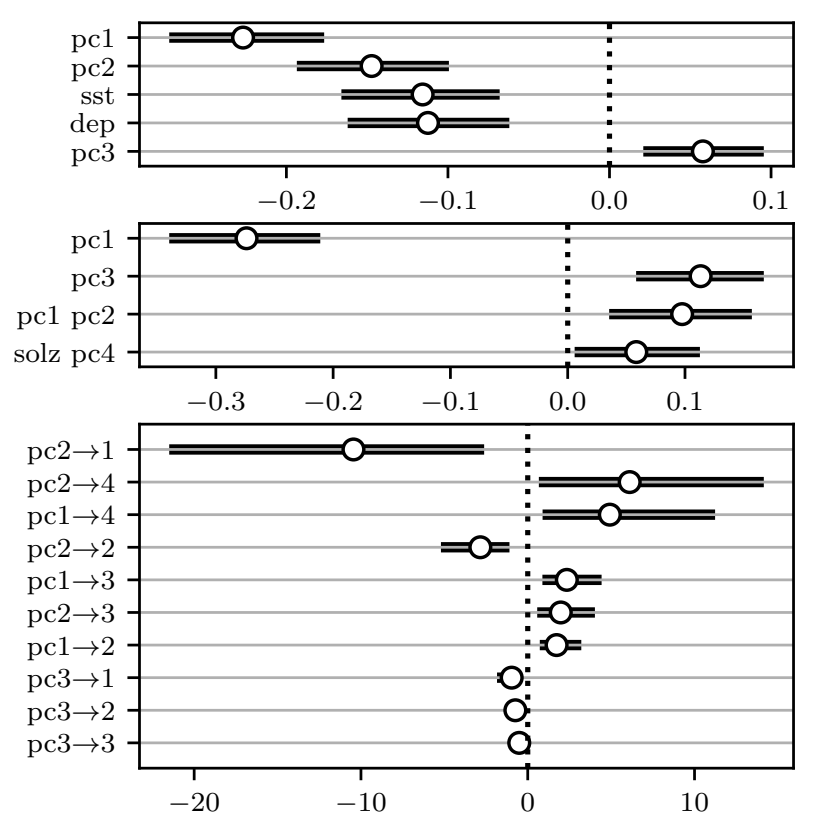

Fig. 1. Forest plots for 3 Bayesian models of $a_{p h}$ (411); top - linear regression; middle - linear regression with interactions; bottom - neural network. Open circles and whiskers are mean and $95 \%$ credibility intervals $(C l)$, respectively, for model coefficients. Each coefficient (shown on the left axes) corresponds to a predictor variable. For brevity, only the most significant predictors (i.e. their $\mathrm{Cls}$ do not overlap the vertical dotted zero line) are shown here in order of descending significance. Predictor abbreviations area as follows; $p c$ - principal component, sst - sea surface temperature, dep - depth, solz - solar zenith angle. Interaction (middle panel) is shown as two predictors side by side. Bottom panel shows predictor connection to neural network hidden layer unit.

tically complex coastal waters using Rayleigh-corrected Topof-Atmosphere reflectance as principal input to a number of models. Of these, Bayesian neural networks resulted in the most robust predictions. This is of particular significance as the information contained in remotely sensed signals of such waters is often irretrievably lost due to failure of atmospheric correction and/or retrieval algorithms. To date, Bayesian approaches have not been used for information retrieval from ocean color and offer a viable alternative to traditional radiative transfer and semi-analytical models. Additionally, Bayesian models provide uncertainty estimates due to their probabilistic nature - something that must be done post hoc if using conventional approaches. The Bayesian framework allows all information concerning uncertainty to be included via formulation of the prior distributions - i.e. environmental, instrumental, systematic uncertainty can all be included in the model if that information is available. This provides a fundamental improvement in how ocean color signals can be used to robustly describe highly dynamic aquatic ecosystems.

Another important feature of this approach is the ability to bypass conventional atmospheric correction techniques. In coastal, inland waters and other optically complex scenarios, it is challenging to accurately achieve atmospheric correction due to the complex assemblage of aerosols that must be modeled and/or the non-zero water leaving radiance that violates fundamental AC model assumptions. In this approach, we do not attempt to subtract the atmospheric contribution, but instead use principal component analysis to 'tease out' the
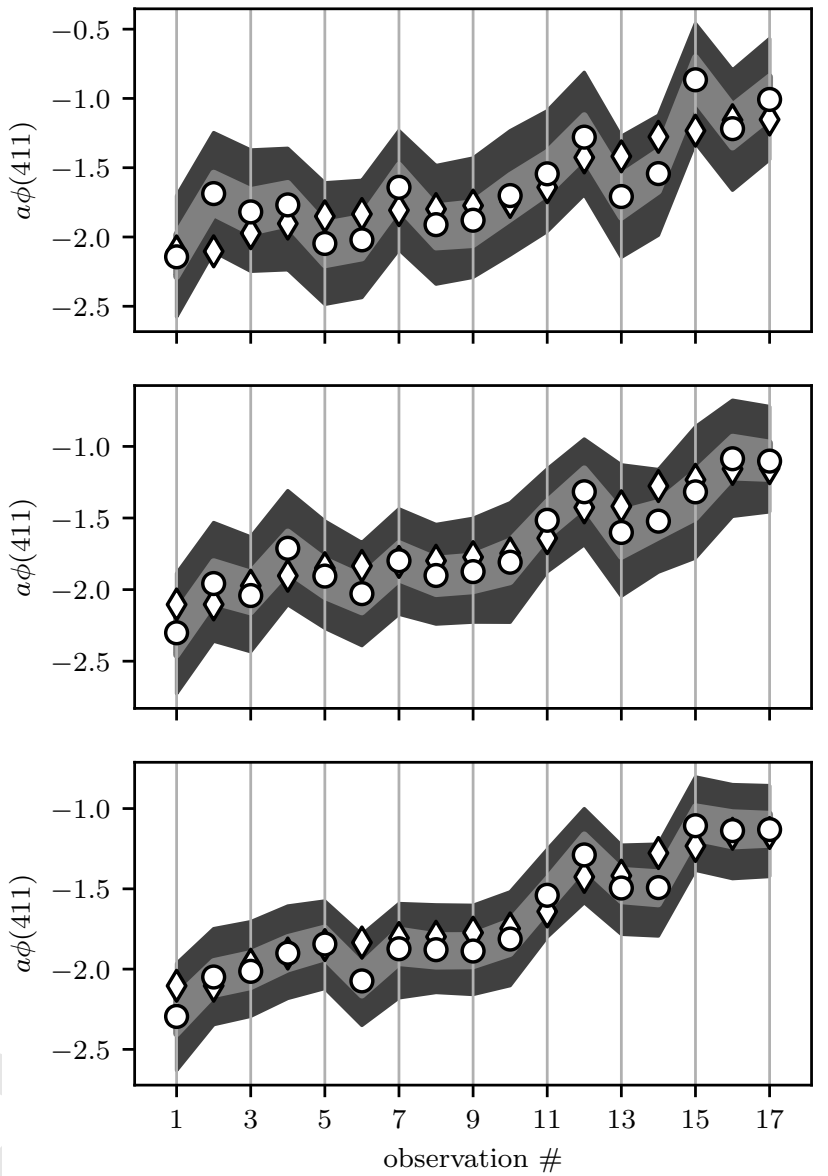

Fig. 2. Posterior predictive plots of out-of-sample $a_{p h}(411)$ data for linear regression (top), linear regression with interaction (middle), neural network (bottom). Y-axes are log scaled. Open diamond $\rightarrow$ out-of-sample observation, arranged monotonically in ascending order; open circle $\rightarrow$ mean model prediction; light gray envelope $\rightarrow 50 \%$ credibility interval $(\mathrm{Cl})$; dark gray envelope $\rightarrow 95 \% \mathrm{Cl}$.

lower modes of variance underlying the large atmospheric signal detected by the satellite sensor. In so doing, we assume nothing regarding the atmospheric scattering or absorbing properties and simply allow the orthogonality conditions of PCA to detect the underlying signals associated with the water and its constituents. Traditional approaches for AC in optically complex waters typically struggle with negative water leaving radiances at the blue end of the spectrum, i.e. too much radiance at the blue end of the spectrum is attributed to the atmosphere by the models used, resulting in a nonphysical, negative water-leaving signal. Our approach is able to completely circumvent this problem and retrieve $a_{p h}(\lambda)$ in the blue with high accuracy.

\section{Materials and methods}

Data collection. A modified version of the NOMAD (Werdell and Bailey 2005) SeaWiFS satellite-to-in situ validation dataset was used for model development (http://seabass.gsfc.nasa.gov/). This was comprised of a subset of the NOMAD dataset limited to stations with coincident and valid SeaWiFS coverage (Fig. 6), and included $R_{r s}$ and TOA radiance $\left(L_{t}(\lambda) ; \mu W \mathrm{~cm}^{-2} \mathrm{~nm}^{-1} s r^{-1}\right)$ in addition to 


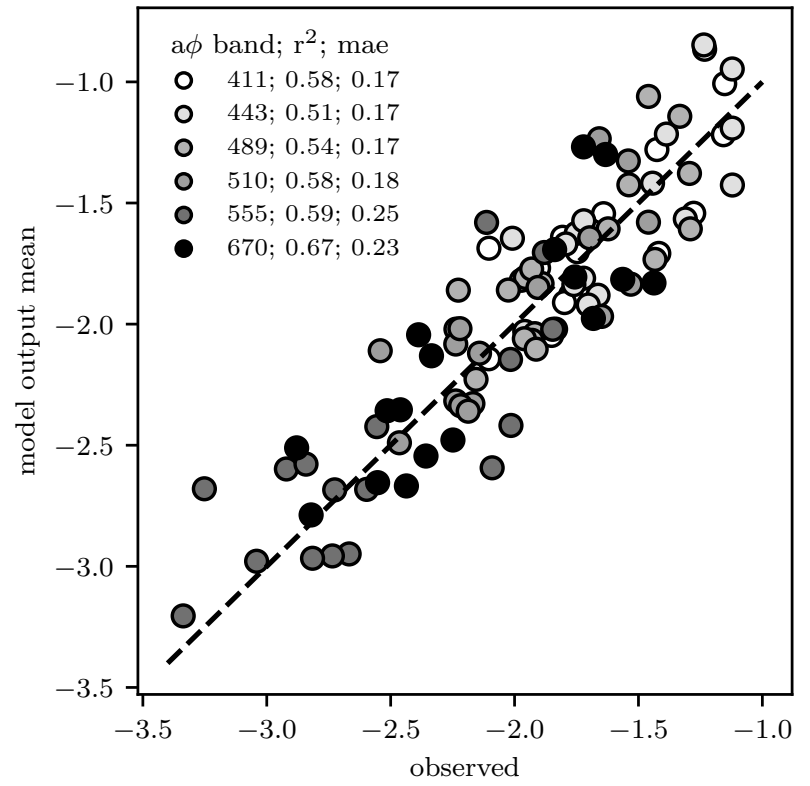

Fig. 3. Out-of-sample observed vs. prediction mean from linear regression for $a \phi$ at 6 bands, featuring $r^{2}$ and mean absolute error (mae) as goodness-of-fit measures. Both axes are log-scaled

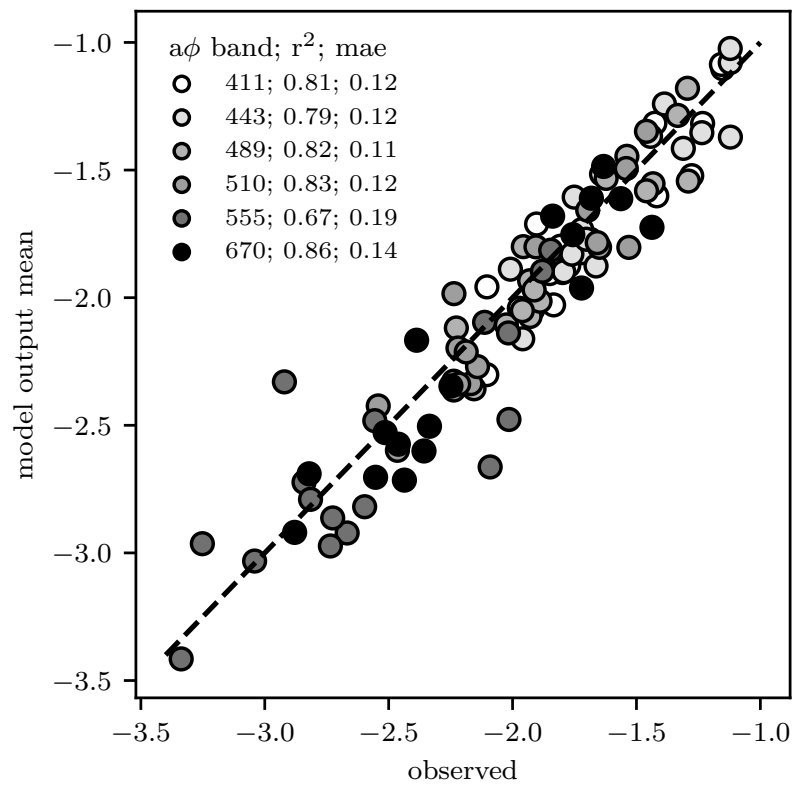

Fig. 4. Out-of-sample observed vs. prediction mean from linear regression with interactions for $\mathrm{a} \phi$ at 6 bands, featuring $r^{2}$ and mean absolute error (mae) as goodness-of-fit measures. Both axes are log-scaled the standard NOMAD parameters such as location, sea surface temperature, water column depth,and solar zenith angle. Rayleigh-corrected remote sensing reflectance $\left(R_{r c} ; s r^{-1}\right)$ was derived using SeaDAS (version 6.2) assuming no aerosol, and is given by:

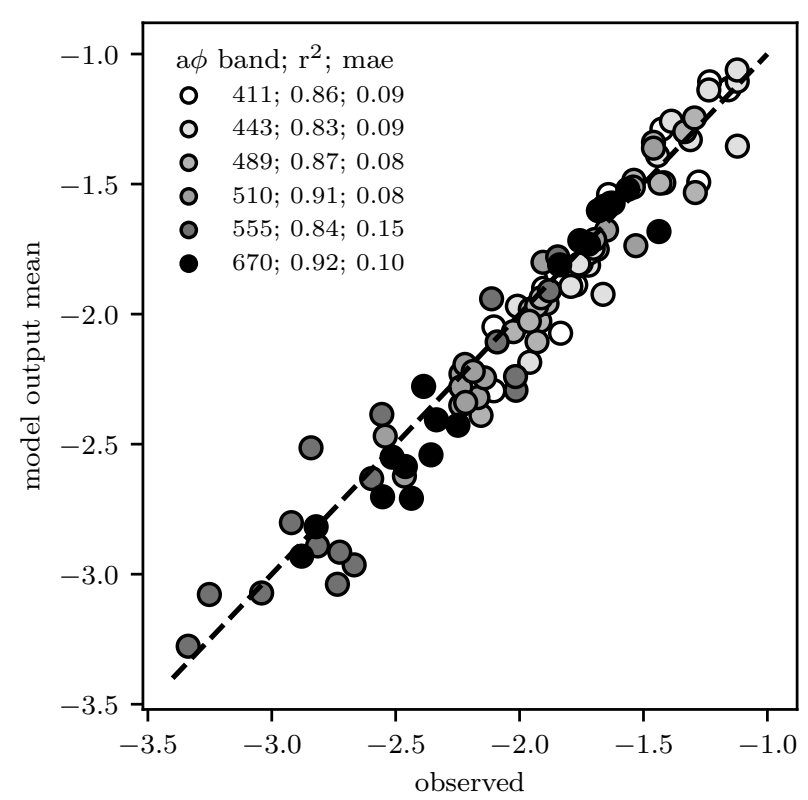

Fig. 5. Out-of-sample observed vs. prediction mean from neural network with interactions for $\mathrm{a} \phi$ at 6 bands, featuring $r^{2}$ and mean absolute error (mae) as goodness-of-fit measures. Both axes are log-scaled

$$
R_{r c}(\lambda)=\frac{L_{t}(\lambda)-L_{r}(\lambda)}{F_{0} \cos \left(\theta_{0}\right) t t_{0}}
$$

where $L_{r}$ is the Rayleigh scattering radiance ${ }_{264}$ $\left(\mu W \mathrm{Cm}^{-2} \mathrm{~nm}^{-1} \mathrm{sr}^{-1}\right), \quad F_{0}$ the extraterrestrial solar irra- 265 diance $\left(\mu \mathrm{Wcm}^{-2} \mathrm{~nm}^{-1}\right), \theta_{0}$ is the solar zenith angle (degrees), $\quad 266$ $t$ the diffuse transmittance from the satellite pixel to the satellite (dimensionless) and $t_{0}$ the diffuse transmittance from the sun to the pixel (dimensionless).

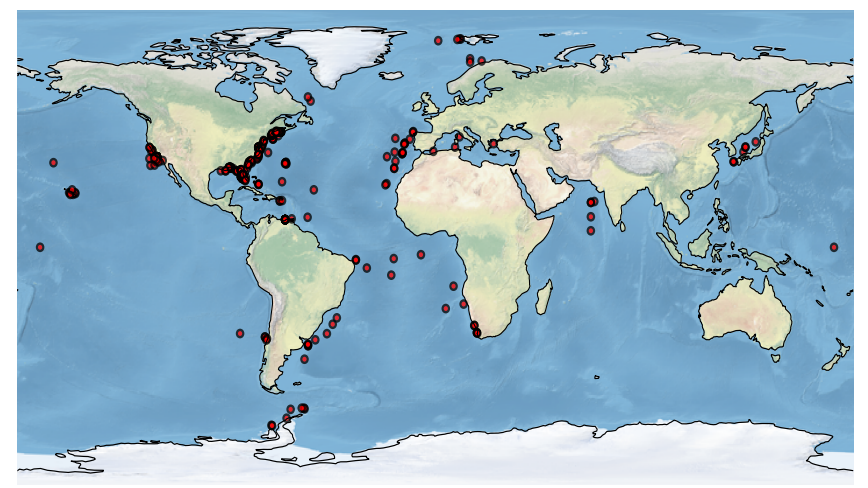

Fig. 6. In-situ sampling stations used in this study.

Data pre-processing. The NOMAD in situ $a_{p h}(\lambda)$ data was provided at 20 wavelengths. This was reduced to 6 to match SeaWiFS visible wavelengths of 412, 443, 490, 510, 555, 670 nm. Data points were discarded if no in situ $a_{p h}(\lambda)$ data existed or had missing wavelengths, and if any of the satellite wavelengths were missing or contained zero values. Three additional pre-processing steps were performed: i) The principal 


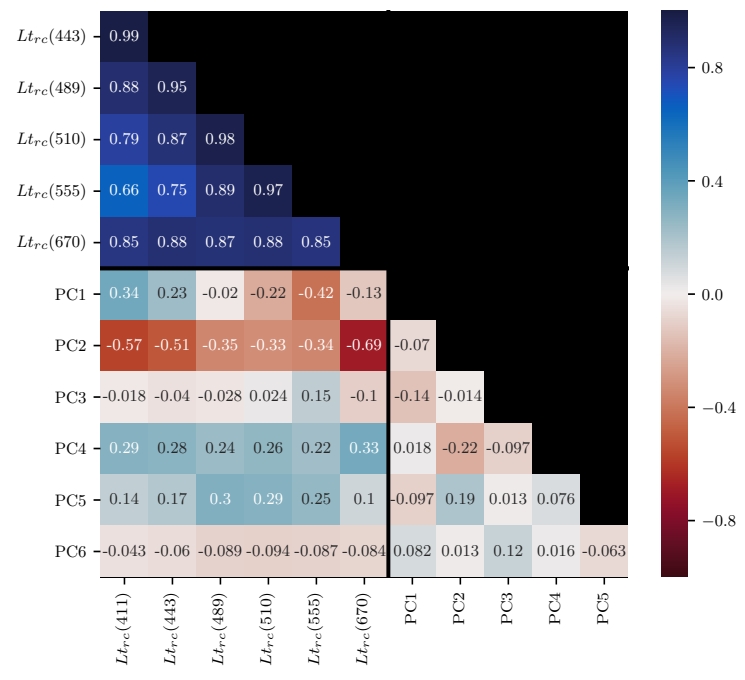

Fig. 7. Pairwise correlation heatmap. Top left quadrant suggests the multicollinearity within TOA radiances $\left(L_{t}\right)$, bottom left quadrant illustrates the link between radiances and principal components.

components (PCs) of the 6 Rayleigh-corrected remote sensing reflectances were computed. After initial model trials, it was found that the PCs were consistently more powerful predictors than the parent reflectance spectra, in agreement with the findings of Craig et al. (16) who observed that $R_{r s}$ PCs were more important predictors of IOPs than reflectance. Using the PCs also has the advantage of eliminating the multicollinearity that exists between the reflectance wavebands (see Fig. 7). This is desirable since inclusion of multiple predictor variables that carry redundant information can introduce model non-identifiability - i.e. the inability to distinguish amongst explanations - that manifests itself by an apparent lack or weak relationship between predictors and predicted variables, when in fact the relationship is much stronger. ii) The data, which included sea surface temperature, solar zenith angle, and reflectance principal components, span widely varying scales. Therefore, they were standardized by subtracting the mean from each predictor variable and dividing by its respective standard deviation. iii) The data was split into training and testing sets, with the training set used for model fitting, while the testing (i.e., out-of-sample) set was used for model predictive skill evaluation.

Model development and fitting. All models described were developed in the Python language using the probabilistic programming library PyMC3(41). Bayesian models to predict the spectral phytoplankton absorption coefficient, $a_{p h}(\lambda)$, were developed. By definition, Bayesian model parameters and their resulting predictions are probabilistic in nature. In brief, Bayes' rule provides a way to update beliefs based on the arrival of new, relevant pieces of evidence, and is expressed as:

$$
P(A \mid B)=\frac{P(B \mid A) P(A)}{P(B)}
$$

where $A$ is the event we want the probability of, and $B$ is the new evidence that is related to $A$ in some way. $P(A \mid B)$ is the posterior; this is what we are estimating. $P(B \mid A)$ is referred to as the likelihood, and is the probability of observing the new evidence, given our initial hypothesis. $P(A)$ is the prior, i.e. the probability of the hypothesis without any additional prior information. $P(B)$ is called the marginal likelihood and is the total probability of observing the evidence.

Each model parameter is initially assigned a prior probability distribution, $P(A)$. The parameter space is then sampled and the likelihood of each observation conditioned on the parameter values is computed at each iteration. Using Bayes rule, the priors are combined with the likelihood to yield a posterior distribution, which quantifies the model parameters' probability given the observations used during sampling. These priors are then updated as the model is confronted with data, through the computation of the model's likelihood for each observation as the parameter space is sampled.

We developed three Bayesian models: linear regression, linear regression with first degree feature interaction terms, and a neural network. Because it was not expected that all predictor variables would be relevant in predicting $a_{p h}$, all models were designed to identify predictor variables that had the greatest impact on the fit. In the case of the linear regression models, this was achieved using a regularized horseshoe prior(42). Regularized horseshoe priors are so called because of the horseshoe shape of the distribution. This shape results from the assignment of high probability both around 0 and far from 0 , and low probability for intermediate values. The assignment of high probability near 0 is not unlike other sparse (where only a subset of predictors is relevant) regression model priors such as those used in Bayesian Lasso and Ridge regression models, in that they assume a number of the the model parameters will effectively shrink to 0 . The horseshoe prior holds a significant advantage over Lasso or Ridge regression priors in that it assigns high probability to 0 while providing a thick tail, which reduces bias. The regularized horseshoe has the advantage that it provides a way to adjust the shrinking rate of non-zero parameters, thereby preventing the model from overfitting on the features corresponding to these nonzero parameters. Fig. 8 (top panel) shows the structure of the linear regression models, with a common intercept parameter given a Gaussian prior, the predictor slope parameters are also Gaussians with the scale parameter $\sigma_{\beta}$ inherited from a combination of 3 hyperpriors (a hyperprior is an assumption made about a parameter in a prior probability assumption) as specified in (42). The linear regression equation is used as the mean of a Gaussian likelihood, which has a standard deviation with a half-Cauchy prior.

Similarly, the Bayesian neural network's construction features automatic relevance determination (ARD)(43). The neural network is fully connected and features one hidden layer. The weights between the input layer and the hidden layer have Gaussian priors as in the linear regression models. However, the spreads of priors for the weights corresponding to each predictor variable have independent half-Cauchy hyper-priors; this is the basis for ARD. On the other hand, the weights connecting the hidden layer to the output layer have Gaussian priors with a common hyperprior for their spread. The Bayesian neural network's architecture is depicted in Fig. 8, bottom panel.

All models were fit using the No U-Turn Sampler, a variant of Hamiltonian Monte-Carlo (44). For the regression models, 2000 samples were drawn after a tuning period made up of 


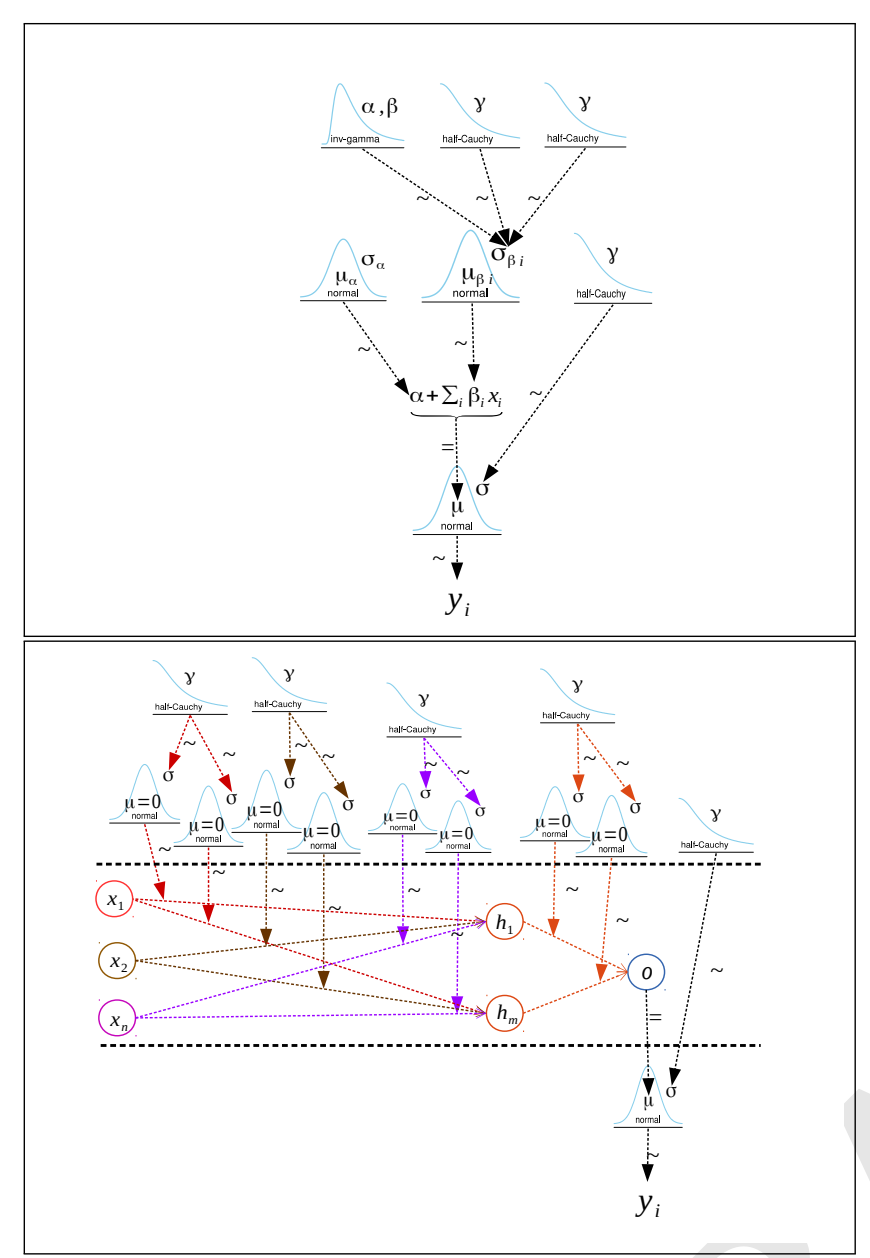

Fig. 8. Inference diagram of Bayesian models used. Horizontal lines separate three conceptual groups; top $\rightarrow$ priors, middle $\rightarrow$ likelihood, bottom $\rightarrow$ outcome distribution Top: Regression with horseshoe priors (Models $1 \& 2$ ). Bottom: Bayesian neural network (Model 3). Models shown here are hierarchical, built for automatic feature relevance determination. exchangeability problem.

Reproducibility. The code describing the preparation and transformation of data, as well as the code for the development, fitting, and evaluation of the models are available through github https://github.com/madHatter106/Bayesian$M L$-4-IOP-from-TOA. The raw data is available through our project page on the Open Science Foundation website $O S F$ link.

\section{Acknowledgments}

\section{References}

1. Hovis WA, et al. (1980) Nimbus-7 coastal zone color scanner: System description and initial imagery. Science 210(4465):60-63.

2. O'Reilly JE, Werdell PJ (2019) Chlorophyll algorithms for ocean color sensors - OC4, OC5 \& OC6. Remote Sensing of Environment 229:32-47.

3. Werdell P, et al. (2018) An overview of approaches and challenges for retrieving marine inherent optical properties from ocean color remote sensing. Progress in Oceanography 160:186212.

4. Behrenfeld MJ, et al. (2006) Climate-driven trends in contemporary ocean productivity. Nature 444:752-755.

5. Saba VS, et al. (2011) An evaluation of ocean color model estimates of marine primary productivity in coastal and pelagic regions across the globe. Biogeosciences 8(2):489-503.

6. Rousseaux CS, Gregg WW (2015) Recent decadal trends in global phytoplankton composition. Global Biogeochemical Cycles 29(10):1674-1688.

7. Dutkiewicz S, et al. (2019) Ocean colour signature of climate change. Nature Communications 10(1):578.

8. Gordon HR, Clark DK (1980) Remote sensing optical properties of a stratified ocean: an improved interpretation. Appl. Opt. 19(20):3428-3430.

9. Gordon HR, Wang M (1994) Retrieval of water-leaving radiance and aerosol optical thickness over the oceans with SeaWiFS: a preliminary algorithm. Appl. Opt. 33(3):443-452.

10. Gordon HR, Du T, Zhang T (1997) Remote sensing of ocean color and aerosol properties: resolving the issue of aerosol absorption. Applied Optics 36(33):8670.

11. Morel A, Prieur L (1977) Analysis of variations in ocean color. Limnology and Oceanography 22(4):709-722.

12. Craig S, et al. (2006) Use of hyperspectral remote sensing reflectance for detection and assessment of the harmful alga, Karenia brevis. Applied Optics 45(21):5414-5425.

13. Darecki M, Stramski D (2004) An evaluation of MODIS and SeaWiFS bio-optical algorithms in the Baltic Sea. Remote Sensing of the Environment 89:326-350.

14. Hu C, et al. (2003) Comparison of ship and satellite bio-optical measurements on the continental margin of the NE Gulf of Mexico. International Journal of Remote Sensing 24(13):2597.

15. Mouw C, et al. (2015) Aquatic color radiometry remote sensing of coastal and inland waters: Challenges and recommendations for future satellite missions. Remote Sensing of Environment 160(0):15-30.

16. Craig SE, et al. (2012) Deriving optical metrics of coastal phytoplankton biomass from ocean colour. Remote Sensing of Environment 119:72 - 83

17. Breitburg D, et al. (2018) Declining oxygen in the global ocean and coastal waters. Science 359(6371):eaam7240.

18. Hare JA, et al. (2016) A vulnerability assessment of fish and invertebrates to climate change on the northeast u.s. continental shelf. PLOS ONE 11(2).

19. Bauer JE, et al. (2013) The changing carbon cycle of the coastal ocean. Nature 504(7478):61-70.

20. Mendonça R, et al. (2017) Organic carbon burial in global lakes and reservoirs. Nature Communications 8(1):1694

21. Bianchi TS, et al. (2018) Centers of organic carbon burial and oxidation at the land-ocean interface. Organic Geochemistry 115:138-155.

22. Frouin RJ, et al. (2019) Atmospheric correction of satellite ocean-color imagery during the pace era. Frontiers in Earth Science 7:145.

23. Ibrahim A, Franz BA, Ahmad Z, Bailey SW (2019) Multiband Atmospheric Correction Algorithm for Ocean Color Retrievals. Frontiers in Earth Science 7(116).

24. Chen J, Cui T, Ishizaka J, Lin C (2014) A neural network model for remote sensing of diffuse attenuation coefficient in global oceanic and coastal waters: Exemplifying the applicability of the model to the coastal regions in Eastern China Seas. Remote Sensing of Environment 148:168-177.

25. D'Alimonte D, Zibordi G, Berthon JF, Canuti E, Kajiyama T (2012) Performance and applicability of bio-optical algorithms in different European seas. Remote Sensing of Environment 124:402-412.

26. Doerffer R, Schiller H (2007) The MERIS Case 2 water algorithm. International Journal of Remote Sensing 28(3/4):517-535.

27. Tanaka A, et al. (2004) Development of a neural network algorithm for retrieving concentrations of chlorophyll, suspended matter and yellow substance from radiance data of the ocean color and temperature scanner. Journal of Oceanography 60(3):519-530.

28. Chen J, Quan W, Cui T, Song Q, Lin C (2014) Remote sensing of absorption and scattering coefficient using neural network model: Development, validation, and application. Remote Sensing of Environment 149:213-226.

29. Liu Y, et al. (2016) Application of deep convolutional neural networks for detecting extreme weather in climate datasets. CoRR abs/1605.01156.

30. Ellen JS, Graff CA, Ohman MD (2019) Improving plankton image classification using context metadata. Limnology and Oceanography: Methods 17(8):439-461. 
31. Gelman A, et al. (2013) Bayesian Data Analysis, Third Edition, Chapman \& Hall/CRC Texts in Statistical Science. (Taylor \& Francis).

32. McElreath R (2016) Statistical Rethinking: A Bayesian Course with Examples in R and Stan, Chapman \& Hall/CRC Texts in Statistical Science. (CRC Press).

33. Kruschke JK (2015) Chapter 11 - null hypothesis significance testing in Doing Bayesian Data Analysis (Second Edition), ed. Kruschke JK. (Academic Press, Boston), Second edition edition, pp. $297-333$.

34. Kruschke JK (2015) Chapter 12 - bayesian approaches to testing a point ("null") hypothesis in Doing Bayesian Data Analysis (Second Edition), ed. Kruschke JK. (Academic Press, Boston), Second edition edition, pp. $335-358$.

35. Craig SE (year?) Derivation of inherent optical properties from satellite top of atmosphere measurements in optically complex waters in NASA PACE Annual Science Team Meeting.

36. Roesler CS, Barnard AH (2013) Optical proxy for phytoplankton biomass in the absence of photophysiology: Rethinking the absorption line height. Methods in Oceanography 7(0):7994.

37. Ulitz J, Stramski D, Reynolds RA, Dubranna J (2015) Assessing phytoplankton community composition from hyperspectral measurements of phytoplankton absorption coefficient and remote-sensing reflectance in open-ocean environments. Remote Sensing of Environment 171:58-74.

38. Ciotti AM, Lewis MR, Cullen JJ (2002) Assessment of the relationships between dominant cell size in natural phytoplankton communities and the spectral shape of the absorption coefficient. Limnology and Oceanography 47(2):404-417.

39. Bojinski S, et al. (2014) The concept of essential climate variables in support of climate research, applications, and policy. Bulletin of the American Meteorological Society 95(9):14311443.

40. Miloslavich P, et al. (2018) Essential ocean variables for global sustained observations of biodiversity and ecosystem changes. Global Change Biology 24(6):2416-2433.

41. Salvatier J, Wiecki TV, Fonnesbeck C (2016) Probabilistic programming in python using PyMC3. PeerJ Computer Science 2:e55.

42. Piironen J, Vehtari A (2017) Sparsity information and regularization in the horseshoe and other shrinkage priors. ArXiv e-prints.

43. Neal RM (1996) Bayesian Learning for Neural Networks. (Springer).

44. Hoffman MD, Gelman A (2011) The No-U-Turn Sampler: Adaptively Setting Path Lengths in Hamiltonian Monte Carlo. arXiv e-prints p. arXiv:1111.4246.

45. Gelman A, Rubin DB (1992) Inference from iterative simulation using multiple sequences. Statist. Sci. 7(4):457-472.

46. Pourzanjani AA, Jiang RM, Petzold LR (2017) Improving the Identifiability of Neural Networks for Bayesian Inference. NIPS Workshop on Bayesian Deep Learning. 